misconceptions among WSW regarding their risk status and the services available to them. Sexually transmitted infections (STIs), abnormal cervical cytology, and unplanned pregnancy are conditions which are crucially exacerbated by barriers to engagement with health care.

Aim(s)/objectives To review the recent literature regarding access to sexual health care among WSW, and discuss some of the indicators of sexual ill-health adversely impacted by barriers to such engagement.

Methods Relevant databases (MEDLINE, Embase) were searched using $\mathrm{MeSH}$ terms related to sexual health, engagement with health serves, and WSW.

Results This review demonstrates that WSW experience several barriers to care, including the heteronormative expectations of health professionals. Studies suggest the prevalence of STIs among WSW is comparable to heterosexual women, while the use of barrier protection is limited. Screening uptake for cervical cancer among WSW remains poor. In addition, sexual minority, sexually active young women are more likely to experience an unplanned pregnancy than their heterosexual peers.

Discussion/conclusion The findings with regards to STI risk and unplanned pregnancy highlight the need for targeted interventions to address sexual risk taking behaviour among WSW. Further research should be conducted to examine the effectiveness of such interventions. Furthermore, greater understanding of the sexual health of WSW is urgently required among clinicians to improve care and engagement with healthcare among this population of women.

\section{P010 MAXIMISING DEPARTMENTAL INCOME; A QUALITY IMPROVEMENT PROJECT FOR IMPROVING HIV TESTING AND CODING}

Laura Parry. Barts NHS Trust, London, UK

\subsection{6/sextrans-2016-052718.65}

Background BASHH standards recommend that $97 \%$ of $1^{\text {st }}$ attenders to GUM services should be offered an HIV test to facilitate prompt diagnosis. This standard now features as a key performance indicator (KPI) in contracts where financial penalties are imposed for non-compliance.

Aim 97\% of first GUM attendances will be offered an HIV test by August 2015.

Methods Quality improvement (QI) methodology was applied and key drivers were identified: 1) Staff: Timetabled administration sessions and training. 2) Communication: Weekly email reminders to staff regarding coding accuracy. 3) Timing: Timely upload of missed HIV codes by reception. 4) Measurement: Performance recorded/reviewed monthly.

Plan-do-study-act cycles (PDSA) were used PDSA 1: Computerised administration recall system launched resulting in all clinical administration tasks becoming computerised and accessible from any site across the Trust. Standard Operating Procedures (SOP) developed. Team training. PDSA 2: Reception team briefed/delegated task of uploading missed HIV codes. Weekly email reminders sent to staff. PDSA 3: Administration recall SOPs uploaded to intranet. New staff inductions delivered.

Results Prior to introduction of this project only $89 \%$ of new attenders were offered an HIV test (May 2014). We have exceeded our aim with $100 \%$ offered, avoiding a potential penalty of $£ 19,165$ per month, securing $£ 229,980$ income over the past 12 months.

Discussion Using QI methodology, robust systems can be implemented improving patient care and facilitating meeting KPIs.

\section{P011 IMPROVING TIME TO TREATMENT; A QUALITY IMPROVEMENT PROJECT FOR RESULTS HANDLING OF NON-STANDARD GUM TESTS}

Laura Parry. Barts NHS Trust, London, UK

\subsection{6/sextrans-2016-052718.66}

Background Delays to treatment following late non-standard results (NSR) review (e.g. mid-stream urine or radiological tests) by a doctor can cause patient harm. There are on average 10 NSR per week in our department. Prior to this project there was limited governance around clinician review of results with most done in an adhoc way sometimes causing significant delays to treatment $(2+$ weeks). Verbal communication with staff often did not result in NSR being actioned faster. Patients would often make multiple calls to the results team resulting in poor patient experience.

Aim All NSR, once available, will be actioned within 7 days by August 2015.

Methods Quality improvement (QI) methodology applied and key drivers identified: 1) Staff: Training, timetabled administration sessions. 2) Communication: Clear roles/responsibilities identified, email communication. 3) Timing: Timely upload of NSR onto recall list by results team. 4) Measurement: Recall list checked daily, NSR remaining recorded.

Plan-do-study-act cycles (PDSA) were used over six months PDSA 1: Developed a computerised recall system. Standard Operating Procedures (SOP) written. Team training. PDSA 2: Results team briefed/delegated task of recording remaining NSR. PDSA 3: SOPs uploaded to intranet. Email communication with new staff. SHO induction briefing (every four months).

Results We now have on average only one outstanding NSR per week. Verbal communication from the results team has confirmed much improved patient satisfaction.

Discussion Through QI methodology and the development of a simple organised governance system, patient care and satisfaction can be improved. Additional PDSA cycles are planned to further service improvement.

\section{P012 MANAGING MYCOPLASMA GENITALIUM: ARE WE DOING ENOUGH?}

${ }^{1}$ Helen Callaby, ${ }^{2}$ Nicolas Pinto-Sander*, ${ }^{2}$ Suneeta Soni, ${ }^{2}$ Daniel Richardson. ${ }^{1}$ Brighton and Sussex Medical School, Brighton, UK; ${ }^{2}$ Brighton ans Sussex University Hospitals NHS Trust, Brighton, UK

\subsection{6/sextrans-2016-052718.67}

Background Mycoplasma genitalium (MG) caus+es urethritis in males and cervicitis and PID in females. MG prevalence in the UK is not well understood and frequent use of single dose macrolide antibiotics is driving antimicrobial resistance.

Methods From November 2011 to May 2015 selected men with persistent urethritis or proctitis and women with persistent PID 
were tested for MG using the Fast-track Diagnostics ${ }^{\mathrm{TM}}$ urethritis PCR.

Results 461 patients were tested for MG. 30/461 (6.5\%) were positive. Median age was 30 years(range 16-53) and more MG-positive males (26/30) than females (4/30) were identified. 1/4 females provided a cervical sample and 3/4 vaginal swabs. Of males, $1 / 26$ provided a penile swab, 3/26 rectal swabs, and 22/26 (84.6\%) gave urine samples. All females self-identified as heterosexual. 10/26 (38\%) men self-identified as men who have sex with men (MSM); 6/30 (20\%) patients were known to be HIV-positive, all of whom were male and 5/6 (83\%) were MSM. 9/30 (30\%) patients were treated with $1 \mathrm{~g}$ single dose azithromycin and $5 / 30(16.7 \%)$ received a regimen of azithromycin $500 \mathrm{mg}$ stat followed by $250 \mathrm{mg}$ od for 4 days. Tests of cure were done in 13/30 (43.3\%). 4/13 (30.7\%) remained positive and all received moxifloxacin, which was curative.

Conclusion We found $\mathrm{MG}$ in symptomatic patients attending our service. Many patients were treated with single dose azithromycin which may be insufficient to clear infection and lead to acquired resistance. Local protocols for persistent urethritis and PID should include routine testing for MG, and newer and better access to diagnostics are urgently needed to support this.

\section{P013 WHEN IS A HERNIA NOT A HERNIA AND LYMPHOMA NOT LYMPHOMA?}

Matthew Hamill. Berkshire Healthcare NHS Foundation Trust, Slough, UK

\subsection{6/sextrans-2016-052718.68}

Background/introduction Lymphogranuloma vereneum (LGV) is a relatively common cause of proctitis and other gastrointestinal symptoms in men who have sex with men (MSM). Other symptoms and signs may present and unless a careful sexual history is taken STI may not be considered in the differential diagnosis.

Aim(s)/objectives To illustrate the potential for mis/inaccurate diagnosis of groin swellings in sexually active MSM and provide a case that can be used for teaching primary care, surgical, oncology and histopathology colleagues.

Methods We present a case of a 55 year old HIV-infected MSM who presented to surgical colleagues with left groin swelling.

Results The patient underwent open surgery to repair an inguinal hernia. At surgery he was found to have significant inguinal lymphadenopathy. Histopathological analysis at the regional pathology centre identified a B cell lymphoma and referral was made to a haematologist to start anti-cancer therapy. In the interim the patient attended our GUM service, was diagnosed with rectal LGV and treated with antibiotics. His lymphadenopathy resolved and staging CT was negative.

Discussion/conclusion Careful consideration of the differential diagnosis of inguinal swelling should be undertaken and STI excluded prior to general anaesthesia and operative procedures whenever possible. Had this patient not attended his GUM clinic he may have undergone potentially toxic chemotherapy to treat LGV infection. This case serves to illustrate the need for open communication between GUM and other medical colleagues.

\section{P014 AN AUDIT OF PREVENTION OF MOTHER TO CHILD TRANSMISSION SERVICES WITHIN A ANTENATAL CARE FACILITY IN A RURAL HEALTH CLINIC IN SWAZILAND}

Sarah Blacker. University of Birmingham Medical School, Birmingham, UK

\subsection{6/sextrans-2016-052718.69}

Background/introduction Swaziland is recorded to have the world's highest HIV prevalence amongst adults and pregnant women. To address this epidemic Swaziland's Ministry of Health $(\mathrm{MOH})$ has adopted the WHO four pronged approach to reducing new HIV infections in women and children.

Aim(s)/objectives To audit whether prevention of mother to child transmission (PMTCT) services at a rural health clinic in Swaziland meets the $2010 \mathrm{MOH}$ targets.

Methods Retrospective data was collected for all women accessing ANC services at the clinic from 1st Feb to 25th May 2015 analysis was performed using Microsoft Excel 2013

Results 29 women accessed ANC services in this time period, $11(37.9 \%)$ were known HIV positive and a further $4(22.2 \%)$ tested positive at presentation. The clinic achieved a HIV testing rate of $94.4 \%$ (target $100 \%$ ) and a partner testing rate of $11.1 \%$ (target 50\%). 93.3\% (15) of HIV positive women received efficacious antiretroviral therapy (target 97\%) and 93.3\% (15) of exposed infants were initiated on appropriate prophylaxis (target 95\%).

Discussion/conclusion This audit has identified areas where action is required for ANC services at the clinic to meet $\mathrm{MOH}$ targets. Early HIV diagnosis and partner testing must be prioritised to reduce new born infections. Access to necessary treatment should be improved by establishing links to antiretroviral clinics.

\section{P015 BASHH MSM SIG CLINIC SURVEY; TESTING AND VACCINATION}

${ }^{1}$ Dan Clutterbuck*, ${ }^{2} \mathrm{MSM}$ Special Interest Group. ${ }^{1}$ Chalmers Centre, Edinburgh, UK; ${ }^{2}$ BASHH, London, UK

\subsection{6/sextrans-2016-052718.70}

Background/introduction/Aim(s)/objectives Our aim was to investigate practice across the UK in aspects of the clinical care of MSM who are HIV negative or of unknown status where evidence is absent, or guidance varies.

Methods An online questionnaire was drafted by the MSM SIG, tested by BASHH CGC members, revised and distributed to BASHH, FSRH members and CSP audit sites for one month to $31^{\text {st }}$ October 2015.

Results There were 149 complete responses. Only 40\% of respondents had a written protocol or policy on recall for HIV/ STI testing of which $23 \%$ had an automated system to recall patients for testing. 50\% routinely test for HIV at syphilis follow up. 90\% of respondents report using both NAAT and culture for GC in contacts of gonorrhoea and $20 \%$ use both in asymptomatic men. $33 \%$ test anatomical sites according to sexual contact history. Self-taken throat (rectal) swabs for GC/Ct NAAT were used never by $26 \%$ (3\%) and routinely in $18 \%(22.5 \%)$. $100 \%$ routinely test MSM for Hepatitis B exposure and over $50 \%$ for Hepatitis C. $78 \%$ routinely check HepB sAb levels 